Reprinted from The Journal of Chemical Physics, Vol. 21, No. 4, 686-688, April, 1953

Printed in U. S. A.

\title{
Effect of Spectral Line Shape on Apparent Rotational Temperatures of $\mathrm{OH}^{*}$
}

\author{
S. S. PENNER \\ Guggenheim Jet Propulsion Center, California Institute of Technology, Pasadena, California
}

(Received November 30, 1952)

\begin{abstract}
The effect of spectral line shape on apparent rotational temperatures of $\mathrm{OH}$ has been investigated for the $P_{1}$ branch, ${ }^{2} \Sigma \rightarrow 2 \Pi$ transitions, $(0,0)$ band, by treating the ratio of collision half-width to Doppler halfwidth as a variable parameter. The results of calculations for emission experiments, using conventional plots, show a large effect of line shape on apparent temperature. In general, the greater the ratio of collision halfwidth to Doppler half-width, the smaller the distortion of experimental data. The analysis predicts higher apparent rotational temperatures for isothermal systems at reduced pressures than at atmospheric pressures. Although this result is in agreement with experimental observations on flames, it cannot be used as an explanation for the observed data without auxiliary studies proving that distortion of data is of importance in any given case. The two-path method for determining temperatures and emissivities (concentrations) in flames has been extended to spectral lines with combined Doppler- and collision-broadening.
\end{abstract}

\section{INTRODUCTION}

$I^{\mathrm{N}}$ $\mathrm{N}$ a recent publication ${ }^{1}$ we have presented the results of quantitative calculations concerning the distortion of emission and absorption data on $\mathrm{OH}$ for various simplified models of flames. These calculations were carried out for spectral lines with Doppler-broadening and hence the results are applicable only to low pressure flames. Although the quantitative interpretation of experimental studies carried out at atmospheric pressure is severely complicated by temperature gradients both normal to and along the line of sight, a great deal of experimental work has been done on flames of this type. ${ }^{2}$ For this reason it appears to be of interest to extend our calculations to spectral lines with combined Doppler- and collision-broadening. Calculations for isothermal systems in emission are presented in Section II where conventional techniques are used for interpretation of experimental data. The calculations

\footnotetext{
* Supported by the U. S. Office of Naval Research under Contract No. Nonr-220(03), NR 015210.

i S. S. Penner, J. Chem. Phys. 21, 31 (1953).

2 H. P. Broida and G. T. Lalos, J. Chem. Phys. 20, 1466 (1952) and earlier publications referred to in this article.
}

predict lower apparent temperatures at elevated pressures under otherwise comparable experimental conditions. Thus they are in accord with observations reported particularly by Gaydon and Wolfhard. ${ }^{3}$ It is, of course, clear that the mere fact that a transition from pure Doppler-broadening to spectral lines with combined Doppler- and collision-broadening produces lower apparent temperatures in isothermal systems, even for relatively weak self-absorption, does not disprove the reality of observed rotational temperatures in flames. On the other hand, it is also apparent that the seemingly plausible argument that the "observed" increase in rotational temperature of $\mathrm{OH}$ with decreasing pressure proves the reality of the anomalous temperatures is entirely unjustified unless it is accompanied by a quantitative proof that the reduction in $P_{\max } X$ will more than offset the distortion produced by a change of line shape. $\dagger$

${ }^{3}$ A. G. Gaydon and H. G. Wolfhard, Proc. Roy. Soc. (London) A208, 63 (1951) and earlier publications.

$\dagger$ Rough estimates utilizing burner dimensions reported in the literature suggest that the probable values of $P_{\max } X$ in low pressure flames are of the same order of magnitude as in flames burning at atmospheric pressure. 
All of our calculations in Section II refer to the $P_{1}$ branch, ${ }^{2} \Sigma \rightarrow{ }^{2} \Pi$ transitions of $\mathrm{OH},(0,0)$ band. For the sake of brevity we shall speak simply of rotational temperatures for the $P_{1}$ branch.

\section{EMISSION EXPERIMENTS}

In order to extend the calculations presented for spectral lines with Doppler contour ${ }^{1}$ to spectral lines with combined Doppler- and collision-broadening, it is most convenient to use van der Held's curves of growth. ${ }^{4,5}$ The line shape is determined by the parameter

$$
a=\left(b_{N}+b_{C}\right)(\ln 2)^{\frac{1}{2}} / b_{D},
$$

where $b_{N}, b_{C}$, and $b_{D}$ denote, respectively, the natural half-width, the half-width resulting from collision(Lorentz-) broadening, and the half-width produced by Doppler-broadening. We have presented previously a graph for calculating total observable intensities,

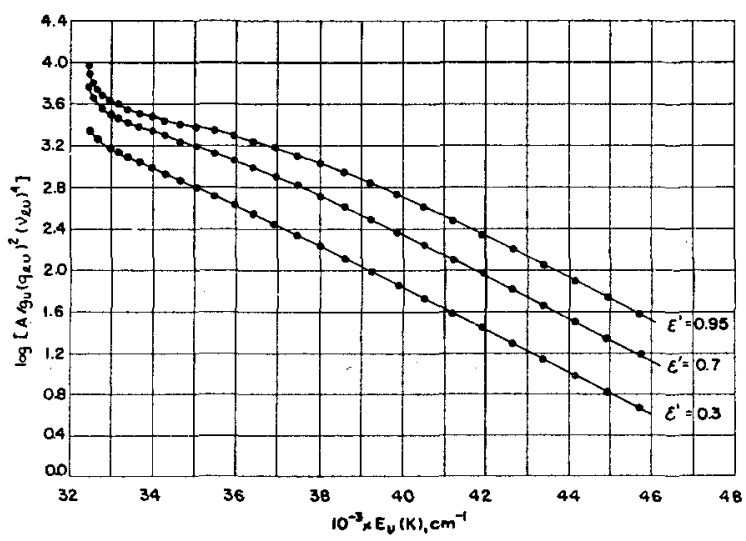

FIG. 1. Conventional plots for the determination of rotational temperatures of $\mathrm{OH}$ in emission for various assumed values of $\epsilon^{\prime}(1)$ for the $P_{1}$ branch, ${ }^{2} \Sigma \rightarrow^{2} \Pi$ transitions, $(0,0)$ band, $T=3000^{\circ} \mathrm{K}$, $a=0.005$.

$A(a=0)$, from peak intensities, $I_{\max }$, for spectral lines with Doppler contour. ${ }^{1}$ For fixed values of the product of maximum absorption coefficient $\left(P_{\max }\right)$ and optical density $(X)$ the ordinate of the curve of growth determines a quantity proportional to the total observable intensity $A(a)$ for arbitrary values of the line contour parameter $a$. Hence, it is a simple matter to obtain, $I_{\max }, A(a=0), A(a) / A(a=0)$ from the curves of growth, and finally absolute values for $A(a)$. Details of the laborious but straightforward calculations will not be presented here.f

The results of numerical calculations for emission

${ }^{4}$ E. M. F. van der Held, Z. Physik 70, 508 (1931); A. Unsöld, Physik der Sternatmosphären, (Edwards Brothers, Inc., Ann Arbor, 1948), p. 168.

${ }^{5}$ For an extension of the curves of growth to larger values of the line-shape parameter $a$, see S. S. Penner and R. W. Kavanagh, Technical Report No. 6, Contract Nonr-220(03), NR 015210 , California Institute of Technology, September, 1952, also J. Opt. Soc. Am. (to be published).

$\ddagger$ The author is indebted to Mr. N. Schroeder for performing the computations.

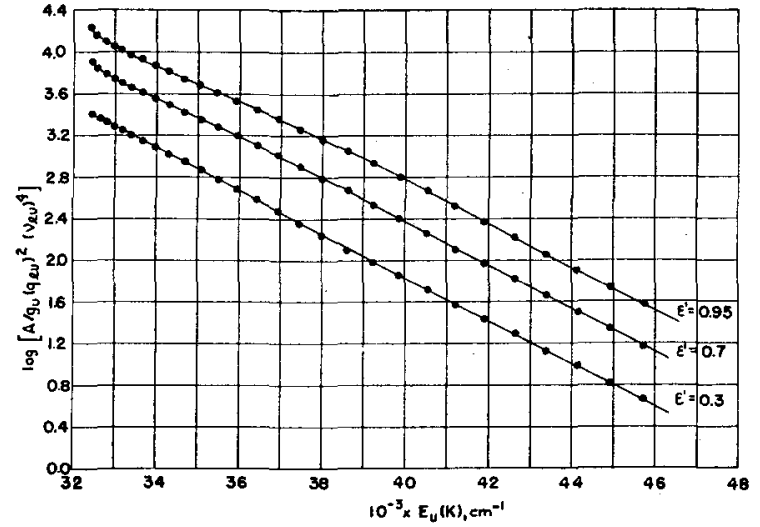

FIG. 2. Conventional plots for the determination of rotational temperatures of $\mathrm{OH}$ in emission for various assumed values of $\epsilon^{\prime}(1)$ for the $P_{1}$ branch, ${ }^{2} \Sigma \rightarrow^{2} \Pi$ transitions, $(0,0)$ band, $T=3000^{\circ} \mathrm{K}$, $a=2$.

from the $P_{1}$ branch at $3000^{\circ} \mathrm{K}$ are summarized by conventional plots for interpretation of data in Figs. 1 and 2 for $a=0.005$ and for $a=2$, respectively. The variable parameter $\epsilon^{\prime}$ is defined in the usual way, viz., $\epsilon^{\prime}=1$ $-\exp \left(-P_{\max } X\right)$, and refers to the first line of the $P_{1}$ branch. ${ }^{1}$ The importance of line shape on the results is evident even from a qualitative comparison between the data plotted in Figs. 1 and 2.

As a rough quantitative measure for the influence of line shape on observable results, we may compute $T_{u}{ }^{\prime}$, the apparent rotational temperature of the upper state, from the calculated data for $10 \leqslant K \leqslant 20$. There is some ambiguity in utilizing this method since the calculated points follow an S-shaped curve for large values of $\epsilon^{\prime}$ (Figs. 1 and 2). The resulting apparent temperatures are summarized in Table I for three values of $a$. The numerical values clearly show that the apparent rotational temperature for isothermal emitters must increase as the pressure is reduced since $a$ decreases as the collision frequency goes down $\left(b_{C}\right.$ is roughly proportional to the pressure and $b_{N} \ll b_{D}$ at the temperatures which are of interest for spectroscopic studies on flames). The effect of line shape, which is expressed quantitatively in Figs. 1 and 2, can be understood by noting that distortion of data by self-absorption must decrease as the effective line width increases, under otherwise comparable conditions.

The extension to spectral lines with combined Doppler- and collision-broadening of our previous studies on absorption experiments, isointensity studies, non-

TABLE I. Effect of line shape and self-absorption on apparent rotational temperatures obtained for $\mathrm{OH}$ in emission experiments ( $P_{1}$ branch of $\mathrm{OH},{ }^{2} \Sigma \longleftarrow_{\pi}$ transitions, $(0,0)$ band, $T=3000^{\circ} \mathrm{K}$, $10 \leqslant K \leqslant 20$ ).

\begin{tabular}{|c|c|c|c|}
\hline$e^{\prime}(1)$ & $a=0.0005$ & $\begin{array}{c}T_{u^{\prime}}\left({ }^{\circ} \mathrm{K}\right) \text { for } \\
a=0.5\end{array}$ & $a=2$ \\
\hline 0.3 & 3410 & 3250 & 3010 \\
\hline 0.7 & 3840 & 3800 & 3230 \\
\hline 0.95 & 5000 & 5000 & 3480 \\
\hline
\end{tabular}




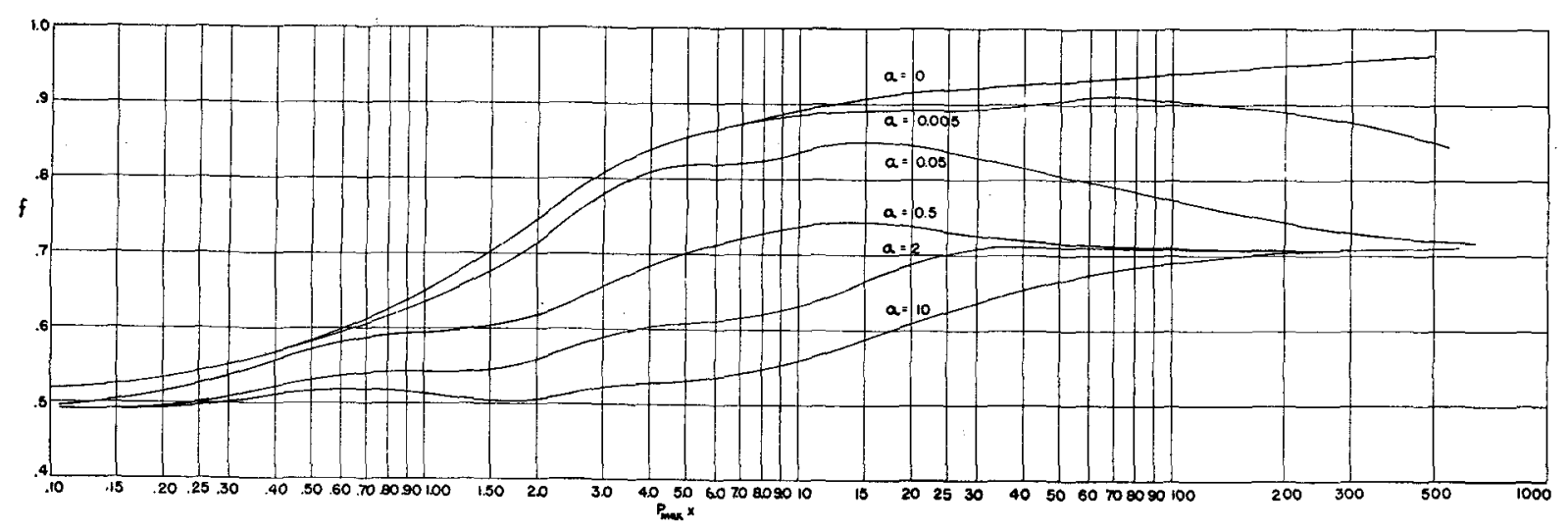

FIg. 3. Plot of observable intensity ratios as a function of $P_{\max } X$ for two-path experiments and various values of the line-shape parameter $a$.

equilibrium mixtures, and nonisothermal systems, as well as calculations for branches other than the $P_{1}$ branch, has also been made but the results will not be presented here. In general, the data are summarized by the remark which has already been made, namely, that distortion of data resulting from self-absorption increases as $a$ decreases. The obvious conclusion which must be drawn from this result is that greater care is required to eliminate self-absorption errors in low pressure flames than in flames burning at atmospheric pressure.

The implications of the result, that apparent rotational temperatures are a sensitive function of line shape (for low resolution spectroscopic studies), are quite disturbing. For example, the dependence of line shape on vibrational quantum number for such molecules as $\mathrm{OH}$ is not known in sufficient detail to rule out the possibility that different apparent temperatures for different branches are produced because of a sizeable difference in the parameter $a$. Other factors may, of course, also be responsible for an effect of this sort.

\section{TWO-PATH EXPERIMENTS FOR ISOTHERMAL SYSTEMS}

The two-path method $^{6}$ for eliminating the effects of self-absorption on experimental data, which permits direct determination both of rotational temperature and of emissivity, has been extended to spectral lines with combined Doppler- and collision-broadening. The calculations can be carried out readily by judicious use of the curves of growth. ${ }^{4,5}$

The results of the laborious calculations are summarized in Fig. 3. The abscissa represents the parameter $P_{\max } X$, and the ordinate $f$ corresponds to the observable ratio of total intensity for the single to the double path. The variable parameter $a$ has been defined previously. For $a=0$ the results apply to spectral lines with pure Doppler-broadening.

Reference to Fig. 3 shows the remarkable fact that the slope of a plot of $f$ is $P_{\max } X$ may change sign repeatedly, particularly for large values of the parameter $a$. This result can be understood by reference to the curves of growth. In so far as applications to interpretation of flame spectra is concerned, the picture evidently becomes complicated for multiple-path experiments on spectral lines with combined Doppler- and collisionbroadening, even if the emitter is isothermal. Details concerning interpretation of flame spectra will appear in a subsequent publication.

\footnotetext{
${ }^{6}$ S. S. Penner, J. Chem. Phys. 20, 1341 (1952).
} 